\title{
Politique
}

\section{La clientèle péquiste en 1985 : caractéristiques et évolution}

\section{André Blais et Jean Crête}

Numéro 10, automne 1986

Opinions et votes

URI : https://id.erudit.org/iderudit/040532ar

DOI : https://doi.org/10.7202/040532ar

Aller au sommaire du numéro

Éditeur(s)

Société québécoise de science politique

ISSN

0711-608X (imprimé)

1918-6584 (numérique)

Découvrir la revue

Citer cet article

Blais, A. \& Crête, J. (1986). La clientèle péquiste en 1985 : caractéristiques et évolution. Politique, (10), 5-29. https://doi.org/10.7202/040532ar d'utilisation que vous pouvez consulter en ligne.

https://apropos.erudit.org/fr/usagers/politique-dutilisation/ 


\title{
LA CLIENTÈLE PÉQUISTE EN 1985: CARACTÉRISTIQUES ET ÉVOLUTION*
}

\author{
André Blais \\ Université de Montréal \\ Jean Crête \\ Université Laval
}

L'élection de novembre 1985 était la cinquième élection à laquelle participait le Parti québécois. Une étude antérieure (Blais et Nadeau, 1984) a tracé l'évolution de la clientèle péquiste depuis la première élection générale à laquelle ce parti avait participé, celle de 1970, jusqu'à celle de 1981. Le présent article vise à déterminer si les caractéristiques socio-économiques des électeurs péquistes ont continué d'évoluer entre 1981 et 1985. Trois éléments de l'analyse de Blais et Nadeau nous intéressent tout particulièrement à cet égard. D'abord, selon ces deux auteurs, le principal clivage électoral au Québec, depuis 1970, en est un de génération, et renvoie essentiellement au rôle des premières expériences électorales de chaque cohorte d'électeurs. Nous examinerons dans quelle mesure le même clivage se manifeste en 1985. Par ailleurs, les

* Cette recherche a été subventionnée par le Conseil de recherche en sciences humaines du Canada et le Fonds FCAR. Les données du sondage nous ont été gracieusement offertes par Le Devoir et la société Radio-Québec. Nous remercions Pierre Mercille pour son assistance dans la préparation des données et Stéphane Dion, François Vaillancourt et les évaluateurs anonymes pour leurs commentaires sur une version antérieure de ce texte. 
trois autres variables qui, de 1970 à 1981, influencaient le plus fortement le vote péquiste (à part la langue) était la pratique religieuse, la syndicalisation et le secteur d'emploi (public ou privé). Il convient de voir ce qu'il en est en 1985, en particulier en ce qui concerne le secteur d'emploi, suite aux coupures de salaires décrétées par le gouvernement péquiste en 1982. Finalement, pour le reste, la clientèle péquiste de 1976 et de 1981 apparaissait de plus en plus indifférenciée sur le plan socio-économique. Nous tenterons d'établir si le processus de diversification s'est maintenu en 1985 .

Les données proviennent du sondage Jolicœur - Le Devoir - Radio-Québec ${ }^{1}$. Les entrevues téléphoniques ont été effectuées du 6 au 18 novembre 1985 et l'échantillon a été tiré à l'aide d'une méthode de génération aléatoire des numéros téléphoniques. Le taux de réponse a été de $63.5 \%$ et le nombre total d'entrevues, $1843^{2}$. Le pourcentage de discrets (indécis et refus) à la question portant sur le vote est de $17 \%$.

L'analyse procèdera à partir de tableaux croisés et de la technique PROBIT, qui permet de mesurer l'effet spécifique d'un certain nombre de variables indépendantes sur une variable dépendante dichotomique (Maddala, 1983; Aldrick et Nelson, 1984). Les coefficients obtenus ont été transformés, afin de pouvoir les interpréter sous forme de probabilités conditionnelles; les coefficients quantifient l'augmentation (ou la diminution) de la probabilité du vote péquiste quand on passe d'une catégorie de la variable indépendante à l'autre, lorsque toutes les autres variables sont gardées constantes.

1. Pour de plus amples informations, voir Le Devoir, 22 novembre 1985.

2. L'échantillon comprend un échantillon initial de 1598 personnes plus un échantillon supplémentaire de 245 non-francophones. Les données utilisées ici ont été pondérées en fonction de 1598 cas. 
Des personnes qui ont révélé leur intention de vote 3 , $41 \%$ ont indiqué leur préférence pour le Parti québécois; le vote péquiste est ainsi surestimé de trois pourcent. L'écart entre le sondage et le vote peut être attribué en partie à un léger déclin du Parti québécois en fin de campagne et en partie à une surreprésentation des jeunes dans l'échantillon (Blais, Crête et Lachapelle, 1986). Le principal déterminant du vote péquiste est évidemment la langue, $49 \%$ des francophones contre $8 \%$ des anglophones et $17 \%$ des allophones disent vouloir appuyer le Parti québécois (tableau 1). Comme le vote péquiste a une signification tout à fait différente chez les francophones et les non-francophones (Hamilton et Pinard, 1976; Blais et Nadeau, 1984), l'analyse doit spécifier la composition de la clientèle dans chacune de ces deux populations.

\section{Le vote des francophones}

Un peu moins de la moitié des francophones a voté pour le Parti québécois. Précisons que les données portent ici sur les individus dont la langue maternelle et d'usage (à la maison) est le français ${ }^{4}$. Le tableau 2 indique l'effet spécifique, toutes choses étant égales par ailleurs, de treize variables socio-économiques sur la probabilité du vote péquiste en $1985^{5}$; seulement trois

3. Sont ainsi exclus les discrets, c'est-à-dire ceux qui se disent indécis ou qui refusent de dire pour quel parti ils ont l'intention de voter, de même que ceux qui veulent s'abstenir ou annuler leur vote. À moins d'indication contraire, cette exclusion s'applique à toutes les données présentées dans ce texte.

4. Seulement 4 des 19 répondants «indiscrets» dont la langue maternelle est le français mais la langue d'usage est autre avaient l'intention de voter pour le Parti québécois.

5. Il est à remarquer que les analyses de régression (tableaux 2 et 10) excluent les répondants pour lesquels l'information n'est pas disponible pour l'une ou l'autre des variables indépendantes. En conséquence, les individus faisant partie d'un ménage qui n'est pas sur le marché du travail ne sont pas considérées, ce qui a réduit le nombre d'observations. Nous avons également effectué des régressions incluant les ménages hors du marché du travail et les résultats sont fort similaires. 


\section{TABLEAU 1}

Le vote péquiste et la langue maternelle, 1985 .

\begin{tabular}{cccc}
\hline & \multicolumn{3}{c}{ Langue maternelle } \\
\cline { 2 - 4 } Vote & $\begin{array}{c}\text { Francophone } \\
(\%)\end{array}$ & $\begin{array}{c}\text { Anglophone } \\
(\%)\end{array}$ & $\begin{array}{c}\text { Allophone } \\
(\%)\end{array}$ \\
\hline Parti québécois & 48.8 & 8.0 & 17.2 \\
Autres partis & 51.2 & 92.0 & 82.8 \\
$\mathrm{~V}=0.29$ & $\mathrm{~N}=1008$ & $\mathrm{~N}=137$ & $\mathrm{~N}=88$ \\
\hline
\end{tabular}

d'entre elles - la pratique religieuse, le lieu de naissance et l'occupation - s'avèrent significatives au seuil de $95 \%$ et deux autres, la scolarité et la présence d'un conjoint, au seuil de $90 \%$. L'absence de relation entre la grande majorité des variables et le vote est probablement le fait marquant de nos données. La clientèle péquiste est ainsi beaucoup plus diversifiée qu'on le croît généralement. Examinons toutefois de plus près l'influence de certaines de ces variables retenues.

\section{a) la pratique religieuse}

La pratique religieuse est, comme en 1981, fortement associée au vote. Le tableau 3 révèle que ce sont les pratiquants réguliers, ceux qui assistent à des cérémonies religieuses à toutes les semaines, qui se démarquent des autres, les pratiquants irréguliers ne se démarquant guère des non-pratiquants. Le tableau 2 indique que, toutes choses étant égales par ailleurs, la probabilité de voter pour

Par ailleurs un examen des corrélations laisse entrevoir qu'un très faible niveau de multicolinéarité dans ces régressions. On notera cependant que la dichotomisation des variables résume l'information et par conséquent peut estomper le caractère linéaire de certaines relations. 


\section{TABLEAU 2}

Le vote péquiste et les caractéristiques socio-économiques chez les francophones en 1985: estimation probit.

Variables (1)

Région

Lieu de naissance

Langue

Religion

Conjoint

Sexe

Âge

Syndicalisation

Chômage

Secteur d'emploi

Occupation

Revenu (3) $(\log$.)

Scolarité $(\log$.)

Scolarité $(\log$.)

Constante

$\mathbf{N}=610$
Estimation probit (2)

$+0.05(+1.09)$

$+0.26(+2.61)^{* *}$

$+0.06(+1.17)$

$-0.23(-4.41)^{* * *}$

$-0.09(-1.71)^{*}$

$-0.03(-0.71)$

$-0.03(-0.46)$

$+0.07(+1.58)$

$+0.02(+0.35)$

$-0.07(-1.43)$

$+0.10(+2.04)^{* * *}$

$+0.03(+1.30)$

$+0.05(+1.77)^{*}$

$-0.45(-2.67)$

(1) La valeur 1 renvoie aux catégories suivantes: région: Montréal; lieu de naissance: Québec; langue: bilingue; religion: fréquentation de cérémonies religieuses toutes les semaines; conjoint : présence d'un conjoint; sexe: homme; âge : 43 ans et moins ; syndicalisation: l'interviewé ou son conjoint est syndiqué; chômage: l'interviewé ou son conjoint a été en chômage au cours de la dernière année; secteur d'emploi: l'interviewé ou son conjoint est dans le secteur public; occupation: le principal gagne pain du ménage est ouvrier. Le revenu et la scolarité sont traités comme des variables numériques et les coefficients indiquent la différence entre la valeur moyenne et la valeur moyenne plus un écart type.

(2) Il s'agit de l'estimation PROBIT transformée afin de pouvoir l'interpréter en fonction de la probabilité conditionnelle. les valeurs du test " $t$ " figurent entre parenthèses.

(3) Il s'agit du revenu du ménage divisé par le nombre de personnes dans le ménage.

* significatif à $90 \%$

*** significatif à $95 \%$

**** significatif à $99 \%$

le Parti québécois diminue du $23 \%$ lorsqu'on est pratiquant régulier.

La situation n'est guère surprenante, la religion étant l'une des variables les plus déterminantes du comportement électoral 
TABLEAU 3

Le vote péquiste et la pratique religieuse chez les francophones, 1985.

\begin{tabular}{lcccc}
\hline \multicolumn{4}{c}{ Assistance aux cérémonies religieuses } \\
\cline { 2 - 5 } Vote & $\begin{array}{c}\text { Toutes } \\
\text { les semaines } \\
(\%)\end{array}$ & $\begin{array}{c}\text { Tous } \\
\text { les mois } \\
(\%)\end{array}$ & $\begin{array}{c}\text { Rarement } \\
) \%)\end{array}$ & $\begin{array}{c}\text { Jamais } \\
(\%)\end{array}$ \\
\hline Parti québécois & 34.9 & 52.4 & 56.7 & 54.7 \\
Autres partis & 65.1 & 47.6 & 43.3 & 43.5 \\
& $\mathrm{~N}=290$ & $\mathrm{~N}=146$ & $\mathrm{~N}=390$ & $\mathrm{~N}=152$ \\
$\mathrm{~V}=0.19$ & & & & \\
\hline
\end{tabular}

dans un grand nombre de pays (Rose, 1974). Au Canada, «the sharpest group difference in Liberal and Conservative party preference is between Catholics and Protestants» (Johnston, 1985, p. 99). En Colombie-Britannique, ceux qui n'ont pas d'affilisation religieuse sont plus susceptibles d'appuyer le NPD (Blake, 1985, tableau 26, p. 87). Pourquoi en est-il ainsi? La pratique religieuse semble associée au conservatisme et aux attitudes de droite (Percheron, 1982). Dans le cas du Québec, il faut souligner que la pratique religieuse régulière, si elle est minoritaire présentement $(30 \%$ des francophones dans notre échantillon disent assister à des cérémonies religieuses à toutes les semaines), a déjà été une norme bien établie, $88 \%$ des québécois disant être des pratiquants réguliers en 1962 (Pinard et Hamilton, 1984, p. 381). Il se pourrait donc que ce soit les mêmes personnes qui aient remis en question cette norme qui aient retiré leur appui aux partis traditionnels et à la communauté politique canadienne (Gingras et Nevitte, 1983, p. 710). 


\section{b) l'occupation}

Le vote péquiste est relativement similaire dans toutes les catégories d'occupation (tableau 4$)^{6}$. Toutefois, lorsqu'on contrôle toutes les autres variables, il ressort que la probabilité de voter pour le Parti québécois est de $10 \%$ plus élevée chez les ouvriers (tableau 2). Si les ouvriers n'apparaissent pas plus péquistes au tableau 4, c'est qu'ils sont moins scolarisés, moins riches et moins bilingues, caractéristiques qui favorisent (faiblement) le vote péquiste. Lorsque ces variables sont neutralisées, les ouvriers ressortent comme légèrement plus péquistes.

De tels résultats contredisent la thèse présentant le Parti québécois comme le parti d'une nouvelle classe moyenne. Cette thèse, qui n'a vraiment été juste que lors des élections de 1970 et de 1973 et exclusivement à l'extérieur de Montréal (Blais et Nadeau, 1984), est encore plus erronée présentement. Il ne faudrait pas non plus exagérer l'écart entre les ouvriers et les autres oc-

TABLEAU 4

Le vote péquiste et l'occupation chez les francophones, 1985.

\begin{tabular}{|c|c|c|c|c|c|c|}
\hline \multirow[b]{2}{*}{ Vote } & \multicolumn{6}{|c|}{ Occupation } \\
\hline & $\begin{array}{l}\text { Adm. } \\
(\%)\end{array}$ & $\begin{array}{l}\text { Prof. } \\
(\%)\end{array}$ & $\begin{array}{l}\text { Semi- } \\
\text { prof. } \\
(\%)\end{array}$ & $\begin{array}{c}\text { Col } \\
\text { Blanc } \\
(\%)\end{array}$ & $\begin{array}{c}\text { Ouvrier } \\
(\%)\end{array}$ & $\begin{array}{l}\text { Agri- } \\
\text { culteur } \\
(\%)\end{array}$ \\
\hline Parti québécois & 55.5 & 59.1 & 53.8 & 48.2 & 53.2 & 51.9 \\
\hline \multirow[t]{2}{*}{ Autres partis } & 44.5 & 40.9 & 46.2 & 51.8 & 46.8 & 48.1 \\
\hline & $\mathbf{N}=55$ & $\mathbf{N}=91$ & $N=95$ & $N=164$ & $N=198$ & $N=10$ \\
\hline $\mathrm{V}=0.06$ & & & & & & \\
\hline
\end{tabular}

6. Nous avons retenu l'occupation de l'interviewé à moins que celui-ci n'ait pas d'emploi, auquel cas nous avons utilisé l'occupation du conjoint (nous avons également utilisé l'occupation du conjoint si celui-ci avait un emploi à plein temps et le répondant un emploi à temps partiel). 
cupations; l'écart, qui est ici de $10 \%$, passe à $6 \%$ (et n'est pas statistiquement significatif) lorsqu'on exclut le revenu. L'emploi n'influence donc que faiblement le vote.

Par ailleurs, les ménages où l'un ou l'autre conjoint a été en chômage au cours des douze derniers mois ne se distinguent guère des autres ${ }^{7}$ et les différents contrôles ne modifient pas cette situation. L'absence de relation peut surprendre dans la mesure ou plusieurs études ont démontré l'impact négatif du taux de chômage sur la popularité des gouvernements (Nadeau, 1985). Mais en fait cette tendance n'émerge de façon nette qu'au niveau agrégé, c'est-à-dire que le vote pour le parti au pouvoir diminue lorsque le chômage augmente, les pertes se manifestant tout autant chez les non-chômeurs (qui deviennent préoccupés par le problème du chômage) que chez les chômeurs. Sur le plan individuel, il ne semble pas y avoir de différence entre chômeurs et non-chômeurs (voir cependant l'analyse des déplacements, section 3), ce qui correspond bien assez bien aux résultats de Kiewitt (1983), qui ne constate également que des différences très faibles aux ÉtatsUnis (voir également Schulzman et Verba, 1979).

\section{c) le lieu de naissance}

Les francophones nés au Québec (tableau 5) votent plus pour le Parti québécois que ceux nés à l'extérieur du Québec, que ce soit au Canada ou dans un autre pays. Le vote péquiste est moins élevé de $26 \%$ dans ce dernier groupe, même après contrôle de toutes les autres variables. C'est là un facteur fort significatif, qui n'avait pas été pris en considération dans les études antérieures. Il convient toutefois de souligner que le groupe en question (ceux

7. Parmi les ménages où l'un des conjoints a été en chômage au cours de la dernière année $51 \%$ appuient le Parti québécois contre $49 \%$ de ceux qui n'ont pas connu le chômage. Par ailleurs, 57 \% de ceux qui étaient en chômage au moment du sondage avaient l'intention de voter pour le Parti québécois. 
TABLEAU 5

Le vote péquiste

selon le lieu de naissance chez les francophones, 1985.

Lieu de naissance

\begin{tabular}{lccc}
\cline { 2 - 3 } Vote & Québec & $\begin{array}{c}\text { Canada } \\
(\text { extérieur du Québec })\end{array}$ & Extérieur du Canada \\
& $(\%)$ & $(\%)$ & 36.6 \\
\hline Parti québécois & 50.0 & 63.9 & 63.4 \\
Autres partis & 50.0 & $\mathrm{~N}=25$ & $\mathrm{~N}=22$ \\
$\mathrm{~V}=0.06$ & $\mathrm{~N}=939$ & & \\
\hline
\end{tabular}

nés à l'extérieur du Québec) est très petit (5\% des francophones dans notre échantillon).

La raison la plus plausible de cet état de fait pourrait être la plus faible intégration des immigrés au milieu québécois francophone ou encore leur plus grande méfiance vis-à-vis le nationalisme (québécois ou autre). Cette explication n'est cependant que très partiellement confirmée par les données, les personnes nées à l'extérieur du Québec n'étant que légèrement plus fédéralistes (écart, non statistiquement significatif, de $6 \%$ ). Notons, par ailleurs, que les francophones nés à l'extérieur du Québec ont eu davantage tendance à voter pour le Parti libéral, lors de la dernière élection fédérale ( $45 \%$, contre $31 \%$ pour ceux nés au Québec). L'appui traditionnel réservé au Parti libéral du Canada chez les immigrants de même que chez les francophones non-québécois est bien connu (Meisel, 1972) et il est possible que cette allégeance «libérale»se répercute au niveau provincial ${ }^{8}$.

8. $71 \%$ des francophones nés à l'extérieur du Québec ayant voté pour le Parti libéral à l'élection fédérale de 1984 avaient l'intention de voter pour le Parti libéral du Québec. Ces immigrés votent moins pour le Parti québécois mais également pour les tiers partis. C'est donc l'allégeance libérale qui semble être le principal facteur d'explication. 
TABLEAU 6

Le vote péquiste selon l'âge chez les francophones, 1985.

\begin{tabular}{lcccccccc}
\hline \multicolumn{10}{c}{ Âge } \\
\cline { 2 - 8 } & $\begin{array}{c}18 \text { à } 19 \\
\text { ans } \\
\text { Vote }\end{array}$ & $\begin{array}{c}20 \text { à } 29 \\
\text { ans } \\
(\%)\end{array}$ & $\begin{array}{c}30 \text { à } 39 \\
\text { ans } \\
(\%)\end{array}$ & $\begin{array}{c}40 \text { à } 49 \\
\text { ans } \\
(\%)\end{array}$ & $\begin{array}{c}50 \text { à } 59 \\
\text { ans } \\
(\%)\end{array}$ & $\begin{array}{c}60 \text { à } 69 \\
\text { ans } \\
(\%)\end{array}$ & $\begin{array}{c}70 \text { ans } \\
\text { et plus } \\
(\%)\end{array}$ \\
\hline Parti québécois & 47.0 & 60.4 & 48.1 & 47.0 & 42.6 & 35.1 & 22.5 \\
Autres partis & 53.0 & 39.6 & 51.9 & 53.0 & 57.4 & 64.9 & 77.5 \\
& $\mathrm{~N}=51$ & $\mathrm{~N}=299$ & $\mathrm{~N}=264$ & $\mathrm{~N}=168$ & $\mathrm{~N}=103$ & $\mathrm{~N}=70$ & $\mathrm{~N}=28$ \\
$\mathrm{~V}=\mathbf{0 . 1 8}$ & & & & & & & \\
\hline
\end{tabular}

\section{d) la scolarité}

Les plus scolarisés ont tendance à appuyer davantage le Parti québécois (tableau 7), la tendance se renversant cependant légèrement chez les très scolarisés (18 ans et plus). Parce que les écarts sont prononcés au départ et s'aplanissent à mesure que la scolarité augmente, la variable donne les résultats les plus intéressants lorsqu'elle est exprimée en termes logarithmiques. Même là cependant, les différences s'affaiblissent lorsque les autres variables sont tenues constantes, en particulier la pratique religieuse, le

\section{TABLEAU 7}

Le vote péquiste selon la scolarité chez les francophones, 1985.

\begin{tabular}{lcccc}
\hline & \multicolumn{4}{c}{ Années de scolarité } \\
\cline { 2 - 5 } & $\begin{array}{c}0 \text { à } 7 \\
\text { ans } \\
\text { Vote }\end{array}$ & $\begin{array}{c}8 \text { à } 11 \\
\text { ans } \\
(\%)\end{array}$ & $\begin{array}{c}12 \text { à } 15 \\
\text { ans } \\
(\%)\end{array}$ & $\begin{array}{c}16 \text { ans } \\
\text { et plus } \\
(\%)\end{array}$ \\
\hline Parti québécois & 33.4 & 45.6 & 52.4 & 59.9 \\
Autres partis & 66.4 & 54.4 & 47.6 & 40.1 \\
& $\mathrm{~N}=109$ & $\mathrm{~N}=183$ & $\mathrm{~N}=448$ & $\mathrm{~N}=141$ \\
$\mathrm{~V}=0.14$ & & & & \\
\hline
\end{tabular}


revenu et l'emploi et ne sont plus significatives au seuil de $95 \%$. Blais et Nadeau (1984) ont noté que l'effet de la scolarité sur le vote n'est vraiment significatif que chez les jeunes. La même tendance émerge en 1985: des analyses de régression, non présentées ici, indiquent que la scolarité n'augmente la probabilité d'avoir appuyé le Parti québécois (à un niveau de signification de $90 \%$ ) que chez les électeurs âgés de 18 à 43 ans; chez les électeurs de 44 ans et plus, la scolarité ne semble aucunement jouer. L'hypothèse alors avancée, à l'effet que "...le système d'éducation, depuis la Révolution tranquille, a véhiculé des valeurs de changement auxquelles le Parti québécois est généralement associé, alors que ce même système d'éducation véhiculait autrefois des valeurs traditionnelles» (Blais et Nadeau, 1984, p. 191) semble donc toujours tenir.

\section{e) la région}

Le Parti québécois a obtenu 7 pourcent plus de votes chez les francophones montréalais que chez ceux du reste de la province. L'écart est réduit à 5 pourcent et n'est pas statistiquement significatif, lorsque les autres variables sont tenues constantes. Nous avons aussi voulu vérifier si certaines régions spécifiques à l'extérieur de Montréal se démarquaient des autres. Il appert que le vote péquiste est plus faible de six pourcent dans la région de Québec que dans le reste de la province (excluant Montréal) mais les régressions où la variable régionale mettait en opposition Québec et le reste de la province n'ont pas donné des résultats significatifs.

\section{f) l'âge}

Le tableau 6 révèle l'appui obtenu par le Parti québécois dans différents groupes d'âge. Le Parti québécois semble parti-

9. Elles demeurent statistiquement significatives lorsque le revenu est exclu de la régression. 
culièrement populaire chez les francophones dans la vingtaine et impopulaire chez ceux de plus de 60 ans. L'impopularité du Parti québécois chez les personnes âgées ne surprendra personne. Mentionnons toutefois que l'âge de la retraite ne semble pas constituer ici un point de rupture important, contrairement à ce qui avait été observé en 1981, 1980 et 1976 (Blais et Nadeau, 1984). Ce point de rupture se situe maintenant davantage autour de 70 ans. Par ailleurs ces résultats sont cohérents avec ceux qu'obtenaient Lemieux, Gilbert et Blais en 1970 lorsqu'ils écrivaient: «...c'est entre les électeurs de 55 ans et plus et les autres que les différences apparaissent les plus grandes» (61). Quinze ans plus tard, cette marge s'est déplacée aux gens de 70 ans et plus.

La forte popularité du Parti québécois chez les gens dans la vingtaine est plus étonnante. En fait, nous nous attendions à ce que le vote péquiste soit plus faible chez les 18-26 ans, soit chez la génération qui a voté pour la première fois alors que le Parti québécois était au pouvoir; les résultats du référendum de 1980 et de l'élection de 1981 accréditaient amplement cette thèse (Blais et Nadeau, 1984). Or cette cohorte d'électeurs est même plus péquiste que les autres dans notre sondage (le Parti québécois y obtient $58 \%$ des votes). Notons toutefois que dans les quatre autres sondages publiés au cours de la campagne pour lesquels l'information est disponible ${ }^{10}$, le Parti québécois obtient moins de votes chez les 18-24 ans que chez les 25-34 ans.

Il demeure toutefois que les différences entre groupes d'âge, sans être négligeables, sont moins marquées qu'elles ne l'étaient auparavant. Qui plus est, ces différences disparaissent lorsque l'on contrôle l'effet des autres variables (tableau 2), tout particulièrement

10. Il s'agit de deux sondages SORECOM, d'un sondage CROP et d'un sondage IQOP (The Gazette, 25 octobre et 23 novembre 1985, La Presse, 2 novembre 1985 et Le Journal de Montréal, 30 novembre 1985). L'écart moyen entre les 18-24 ans les 25-34 ans dans ces quatre sondages est de $11 \%$ (ces résultats incluent cependant les non-francophones). 
la pratique religieuse, qui est fortement associée à l'âge et au vote $^{11}$.

\section{g) le sexe}

Il n'y a aucune différence entre le comportement des hommes et des femmes et cette absence de différence se maintient dans les données de l'analyse multivariée. En 1981, Blais et Nadeau (1984) avaient noté un écart appréciable entre les hommes et les femmes chez les ménages sans conjoint de la région de Montréal. La même tendance (non statistiquement significative) se manifeste en 1985 , le pourcentage du vote péquiste étant de $12 \%$ plus élevé chez les hommes et cet écart ne disparaît pas lorsqu'on contrôle l'âge. Il convient cependant de conclure que dans l'ensemble le vote ne varie guère en fonction du sexe.

\section{b) le secteur d'emploi}

Les travailleurs du secteur public sont un peu plus (6\%) susceptibles d'avoir voté pour le Parti québécois que ceux du secteur privé. Cet écart est renversé ( $-7 \%$ ), sans être significatif, lorsque les autres variables sont prises en considération, le secteur public étant davantage syndiqué, scolarisé et ayant des revenus plus élevés. Notons par ailleurs que les travailleurs du secteur public provincial ne se démarquent guère de ceux des niveaux municipal ou fédéral. L'absence d'association est en soi significative, puisqu'elle suggère l'existence de déplacement du vote entre 1981 et 1985 , suite aux coupures de salaire effectuées dans le secteur public. Cette question sera examinée plus loin, lorsqu'on étudiera la clientèle qui a quitté le Parti québécois, entre 1981 et 1985.

\section{i) la syndicalisation}

Le vote péquiste est plus élevé de six pourcent dans les ménages qui comptent au moins un syndiqué. L'écart est de sept 
pourcent lorsque toutes les autres variables sont neutralisées. La tendance n'atteint pas le seuil de signification habituel et est donc incertaine. Par ailleurs c'est seulement chez les personnes âgées de l'extérieur de la région de Montréal que la syndicalisation semble jouer un rôle ${ }^{11}$. L'écart entre syndiqués et non syndiqués est de $32 \%$ chez les électeurs de 44 ans et plus de l'extérieur de la région de Montréal; pour la même catégorie d'âge, il est nul pour la région de Montréal et pour les jeunes, quelle que soit la région. L'influence de la syndicalisation chez les personnes "plus âgées» de l'extérieur de la région de Montréal tend à corroborer la thèse selon laquelle les syndicats ont contribué à diffuser le message péquiste dans les milieux qui lui étaient traditionnellement le plus imperméables (Blais et Nadeau, 1984).

\section{j) le revenu}

Il n'y a guère de relation univoque entre le revenu familial et le vote (tableau 8). Nous avons également voulu vérifier l'existence de relations plus complexes, de type logarithmique ou non-linéaire. Nous avons construit d'autres mesures de revenu, soit le revenu par travailleur et le revenu par membre du ménage, pour lesquelles nous avons également introduit des transformations logarithmiques et non-linéaires. Dans aucun cas, l'une ou l'autre des mesures du revenu ne s'est avérée statistiquement significative ${ }^{12}$. On doit donc conclure qu'il ne s'agit pas là d'une variable pertinente.

11. Nous avons opérationnalisé l'âge de différentes façons (différents points de rupture, valeur numérique, transformation logarithmique, valeur au carré...) et dans aucun cas la variable s'est-elle avérée statistiquement significative. Dans le tableau 2, on a retenu le point de coupure jugé le plus pertinent sur le plan théorique, c'est-à-dire les électeurs de 18 à 43 ans, qui ont voté pour la première fois en 1966 ou après. Nos données, prises en coupe instantannée ne permettent pas de différencier les causes (cycles de vie, génération ou période) de cet estompage des différences entre groupes d'âge.

12. Dans l'équation du tableau 2, nous avons retenu celle des mesures qui donnait les résultats les plus intéressants. 
TABLEAU 8

Le vote péquiste selon le revenu familial chez les francophones, 1985.

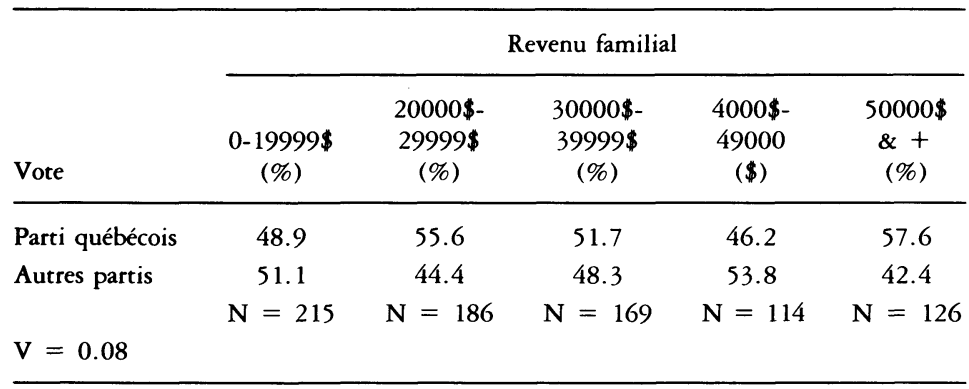

\section{k) la présence d'un conjoint}

Le Parti québécois recueille six pourcent moins de votes chez les personnes demeurant avec un conjoint; l'écart augmente à neuf pour cent lorsqu'on contrôle toutes les autres variables mais n'est significatif qu'au seuil de dix pourcent. Une tendance similaire avait été notée par Hamilton et Pinard (1976) mais ne s'était pas manifestée en 1980 et 1981 (Blais et Nadeau, 1984). Les données sont donc ici particulièrement ambiguës. Une analyse plus fine du vote selon le type de ménage (avec ou sans conjoint, selon que les deux conjoints sont sur le marché du travail ou non...) est présentée ailleurs (Blais et Crête, 1986).

\section{l) le bilinguisme}

Le vote péquiste est plus élevé de huit pourcent chez les francophones qui disent pouvoir soutenir assez bien une conversation en français et en anglais; l'écart diminue (6\%) après contrôle des autres variables et n'est pas significatif au seuil habituel de cinq pourcent. Ce lien entre vote péquiste et bilinguisme a déjà été observé dans des enquêtes antérieures (OLZAK, 1982, p. 269). Il n'en demeure pas moins paradoxal, à première vue, que le 
bilinguisme conduise, si faiblement soit-il, à appuyer un parti souverainiste. L'explication pourrait être que ce sont ces bilingues qui sont le plus directement en contact avec le milieu anglophone et que c'est là une situation propice à l'éclosion du nationalisme. Cette explication va dans le sens de la théorie de la mobilisation ethnique aiguillonnée par la compétition pour les ressources, notamment la compétition pour l'emploi (OLZAK, 1982, 254256). À cet égard, il est intéressant de constater que ce n'est que dans le secteur privé que les bilingues se distinguent des nonbilingues (écart de $8 \%$, significatif à .03).

En somme, la probabilité qu'un électeur ait voté pour le Parti québécois en 1985 est plus grande si cet électeur n'est pas un pratiquant régulier, s'il est né au Québec et s'il est ouvrier. Pour le reste, les relations entre les diverses variables socio-économiques et le vote sont ténues et la clientèle péquiste apparaît fort diversifiée ${ }^{13}$.

\section{Le vote des non-francophones}

Les non-francophones ont peu voté pour le Parti québécois. Dans notre sondage ${ }^{14}$ six pourcent ont dit avoir l'intention d'appuyer le Parti québécois, soit huit pour cent des indiscrets, ce qui représente un recul d'environ $10 \%$ par rapport à 1981 (Blais et Nadeau, 1984). Le petit nombre d'électeurs péquistes $(n=26)$ parmi les non-francophones empêche de procéder à une analyse multivariée et de tirer des conclusions générales. Notons toutefois

13. Un test excluant la variable religion, variable de comportement dont on pourrait contester la présence dans une liste de variables indépendantes, a donné des résultats presqu'identiques à ceux rapportés au tableau 2 sauf pour le coefficient de l'âge qui passe alors de -0.03 à +0.08 mois qui demeure statistiquement non significatif.

14. C'est-à-dire ceux dont ni la langue d'usage ni la langue maternelle n'est le français. Pour cette partie de l'analyse, les non-francophones, qui sont sur-représentés dans l'échantillon, ont été ramenés à leur nombre réel, alors qu'ils ont un poids plus faible dans l'échantillon total (voir Le Devoir, 22 novembre 1985). 
que sur les 26 électeurs péquistes, 18 sont nés à l'extérieur du Québec et 19 disent pouvoir soutenir une conversation en français. Les autres variables ne semblent aucunement jouer. Par exemple, les jeunes, les non-pratiquants, les syndiqués et les plus scolarisés ne sont pas davantage péquistes. Ce serait donc l'intégration à la communauté francophone ou la non-intégration à la communauté anglophone qui expliquerait le comportement marginal de ce petit groupe d'électeurs.

\section{Les déplacements de vote}

Les tendances qui viennent d'être dégagées ont un caractère statique, puisqu'elles décrivent la situation en 1985, sans identifier les changements qui se sont produits entre 1981 et 1985. Et pourtant changements, il y a eu. Dans notre sondage, parmi les électeurs francophones qui ont dit avoir appuyé le Parti québécois en 1981, un bon $29 \%$ n'ont pas voté pour ce parti en 1985 (tableau 9). De même $12 \%$ de ceux qui ont voté pour un autre parti en 1981 se sont ralliés au Parti québécois ${ }^{15}$. Il est donc important d'examiner les caractéristiques de ceux qui ont modifié leur comportement entre 1981 et 1985. Nous allons ici nous intéresser au groupe le plus nombreux, c'est-à-dire les francophones qui ont voté pour le Parti québécois en 1981 et pour un autre parti en $1985^{16}$.

Le tableau 10 indique les résultats de l'analyse PROBIT chez les francophones qui ont voté pour le Parti québécois en 1981 ; il permet d'identifier les caractéristiques des électeurs qui

15. Ces données et toutes celles qui suivent, ne tiennent pas compte des abstentions, à propos desquelles les sondages ne donnent pas des informations assez fiables. Dans notre sondage seulement $3 \%$ des interviewés ont dit avoir l'intention de s'abstenir.

16. Comme nous n'avons que 30 francophones qui ont voté pour le Parti québécois en 1985 et pour un autre parti en 1981 , une analyse systématique de ce type de déplacement s'avère impossible. 
TABLEAU 9

Le comportement électoral de 1985 selon le comportement électoral de 1981 chez les francophones.

\begin{tabular}{lccc}
\hline & \multicolumn{3}{c}{ Vote, 1981 } \\
\cline { 2 - 4 } Vote, 1985 & $\begin{array}{c}\text { Parti québécois } \\
(\%)\end{array}$ & $\begin{array}{c}\text { Parti libéral } \\
(\%)\end{array}$ & $\begin{array}{c}\text { Autres partis } \\
(\%)\end{array}$ \\
\hline Parti québécois & 70.9 & 8.0 & 44.4 \\
Parti libéral & 19.9 & 87.7 & 41.6 \\
Autres partis & 9.1 & 4.3 & 14.0 \\
& $\mathrm{~N}=458$ & $\mathrm{~N}=226$ & $\mathrm{~N}=26$ \\
\hline
\end{tabular}

ont eu davantage (ou moins) propension à rester fidèles au Parti québécois en 1985. Notons dès le départ que les caractéristiques des déplacements sont plus marquées que celles des clientèles en 1985 et qu'on obtient ici de meilleurs résultats. En fait six variables sont significatives au seuil de $5 \%$ et une autre au seuil de $10 \%$. Il ressort que le Parti québécois a perdu davantage d'appui en 1985, à Montréal, chez les électeurs de 43 ans et moins, les employés du secteur public provincial, les non-bilingues, les pratiquants réguliers et les semi-professionnels; ses pertes sont également plus prononcées chez les ménages qui ont connu le chômage.

Le résultat le plus prévisible concerne évidemment les employés du secteur public provincial. Le mécontentement des fonctionnaires, à la suite des coupures de salaires décrétées par le gouvernement en 1982, était bien connu. Nos données indiquent que, tout étant égal par ailleurs, les pertes du Parti québécois sont de $18 \%$ plus élevées chez les fonctionnaires provinciaux. Comme les ménages francophories comprenant au moins un fonctionnaire provincial représentent $15 \%$ de l'échantillon, les pertes subies auprès de ce groupe correspondent à un recul de l'ordre de $3 \%$ du Parti québécois entre 1981 et 1985 . Cela n'est certainement pas négligeable mais n'est cependant pas suffisant pour rendre compte 
TABLEAU 10

Le vote péquiste en 1985 chez les électeurs francophones péquistes de 1981 :

L'influence des caractéristiques socio-économiques; estimation probit.

\begin{tabular}{ll}
\hline Variables (1) & Estimation probit (2) \\
\hline Région & $-0.11(-2.13)^{* *}$ \\
Lieu de naissance & $-0.18(-0.84)$ \\
Langue & $+0.12(+2.16)^{* *}$ \\
Religion & $-0.33(+4.63)^{* * *}$ \\
Conjoint & $-0.01(-0.12)$ \\
Sexe & $-0.06(-1.15)$ \\
Âge & $-0.18(-2.74)^{* * *}$ \\
Syndicalisation & $+0.03(+0.44)$ \\
Chômage & $-0.12(-1.85)^{*}$ \\
Secteur d'emploi & $-0.18(-2.71)^{* * *}$ \\
Occupation & $-0.25(-3.14)^{* * *}$ \\
Revenu (3) (log) & $+0.04(+1.37)$ \\
Scolarité (au carré) & $+0.05(+1.16)$ \\
Constante & $+0.12(+0.22)$ \\
& $\mathrm{N}=345$ \\
\hline
\end{tabular}

(1) La valeur 1 renvoie aux catégories suivantes: région: Montréal; lieu de naissance: Québec; langue: bilingue; religion: fréquentation de cérémonies religieuses toutes les semaines; conjoint: présence d'un conjoint; sexe : homme; âge: 43 ans et moins; syndicalisation: l'interviewé ou son conjoint est syndiqué; chômage: l'interviewé ou son conjoint a été en chômage au cours de la dernière année; secteur d'emploi: l'interviewé ou son conjoint est dans le secteur public provincial; occupation: le principal gagne-pain est semi-professionnel. La scolarité et le revenu sont traités comme des variables numériques et les coefficients indiquent la différence entre la valeur moyenne et la valeur moyenne plus un écart-type.

(2) Il s'agit de l'estimation PROBIT transformée afin de pouvoir l'interpréter en fonction de la probabilité conditionnelle. Les valeurs du test " $t$ " figurent entre parenthèses.

(3) Il s'agit du revenu du ménage divisé par le nombre de personnes dans le ménage.

* significatif à $90 \%$

** significatif à $95 \%$

*** significatif à $99 \%$

de l'essentiel des déplacements du vote. Le secteur d'emploi n'est que l'une des six variables significatives et ce n'est d'ailleurs pas celle qui est la plus significative. Rien n'indique de plus que le mécontentement des fonctionnaires ait eu des effets de contagion 
auprès de l'ensemble des syndiqués, la variable syndicalisation n'étant aucunement associée au déplacement du vote.

Le Parti québécois perd également des appuis chez les électeurs nés après 1943, c'est-à-dire auprès de la génération qui constituait, jusqu'en 1981, le bloc péquiste le plus solide (Blais et Nadeau, 1984). Ces pertes sont telles que les ruptures entre cohortes d'âge, qui s'étaient manifestées nettement depuis 1970, ne se reproduisent guère en 1985. Il resterait à préciser les raisons de cette désaffection. Peut-être, comme le suggère Monière (1985), la déception a-telle gagné cette génération qui, pendant 20 ans, avait misé sur la mobilisation politique. Cette hypothèse n'est cependant pas corroborée par nos données puisque, d'une part, il n'y a pas de signe de dépolitisation massive dans notre sondage ${ }^{17}$ et que d'autre part, le Parti québécois n'a pas perdu davantage de terrain chez les répondants de 43 ans et moins qui ont dit s'intéresser moins à la politique qu'il y a cinq ans ${ }^{18}$. Notons par ailleurs que ce n'est que chez les jeunes du secteur public que le Parti québécois recule.

D'autre part, les pertes péquistes s'avèrent plus prononcées chez les semi-professionnels. En examinant de plus près les données, on constate que ce sont les semi-professionnels du secteur privé qui abandonnent le plus le Parti québécois (notons toutefois que les nombres sont ici petits), aucune tendance n'émergeant chez les employés du secteur public.

Les pertes péquistes sont aussi plus élevées chez les pratiquants réguliers, groupe particulièrement peu péquiste au départ. D'une certaine façon, ce résultat est surprenant. Comme en 1981 le chef du Parti libéral était assez nettement identifié à la religion catholique,

17. $25 \%$ des personnes interviewées ont indiqué que la politique les intéressait moins qu'il y a cinq ans, $35 \%$ qu'elle les intéressait plus et $40 \%$, autant.

18. Notons toutefois que nous ne prenons pas ici en considération les abstentions, pour lesquelles la thèse de Monière pourrait s'avérer plus juste. 
on aurait pu penser qu'il aurait fait le plein du vote des pratiquants réguliers et que le Parti québécois fasse une certaine percée en 1985. Tel n'est manifestement pas le cas. L'hypothèse la plus plausible est que les participants réguliers ont encore plus boudé le Parti québécois en 1985 parce que le gouvernement avait indiqué son intention de déconfessionnaliser la structure du système scolaire ou encore que l'enjeu scolaire, auquel la religion est intimement associée, ait été jugé plus important en 1985 qu'en 1981.

Le Parti québécois a également perdu davantage de terrain chez les non-bilingues. Il convient peut-être de noter qu'en 1985 le chef du Parti québécois n'avait pas un nom français alors que c'était le chef du Parti libéral qui se trouvait dans cette situation en 1981. Et effectivement les unilingues francophones ont nettement moins confiance en $\mathrm{M}$. Johnson que les bilingues (écart de $13 \%$, significatif à $99 \%$ ). Qui plus est, c'est chez ceux qui disent voter d'abord en fonction des chefs que l'écart entre unilingues et bilingues est le plus prononcé (différence de $20 \%$, significative à $99 \%)^{19}$.

D'autre part, on a pu noter que la place de la langue française dans la société québécoise n'était plus l'objet d'un débat lors de l'élection de 1985, contrairement aux élections antérieures. Dans ces circonstances, seuls les électeurs spécifiquement touchés par les problèmes linguistiques - les bilingues, qui sont plus directement en contact avec les milieux anglophones que les unilingues - seraient affectés par cette variable. En d'autres mots, le débat linguistique aurait intéressé une clientèle davantage spécialisée en 1985 qu'en 1981.

19. Ce résultat peut être mis en parallèle avec une explication similaire, suggérée récemment pour rendre compte du succès remporté par des candidats d'extrême droite aux élections primaires de l'Illinois. "Apparently many voters around the state unfamiliar with the candidates, cast ballots for Fairchild and Hart because their names sounded more confortable to them than those of their regular Democratic opponents, George Sangmeister and Aurelia Pulinski» (Time, 31 mars 1986, p. 29).

Nous remercions François Vaillancourt d'avoir porté ce fait à notre attention. 
Le déclin du Parti québécois est par ailleurs plus marqué dans la région de Montréal que dans le reste de la province. La démission de ministres importants du gouvernement dans la région de Montréal (on pense en particulier aux ministres Parizeau et Laurin) n'est probablement pas étrangère à cette situation. Le processus enclenché depuis 1976 (Blais et Nadeau, 1984) est donc pour ainsi dire achevée; du parti essentiellement montréalais qu'il était, il s'est transformé, en moins de dix ans, en un parti tout aussi bien implanté à l'extérieur de Montréal. C'est peut-être là l'une de ses plus grandes réussites.

Finalement, ceux qui ont connu le chômage au cours des douze derniers mois précédant l'élection se sont montrés moins fidèles au Parti québécois. Les chômeurs puniraient ainsi le gouvernement sortant pour une performance économique jugée négative (Nadeau, 1985). Pinard et Hamilton (1973, p. 238) ont noté que le chômage dans la famille immédiate était positivement relié au vote pour l'opposition péquiste en 1973. On y décelait une interaction complexe entre chômage, région et scolarité. Il conviendrait cependant de souligner qu'aux États-Unis les chômeurs ont légèrement plus tendance à voter pour le Parti démocrate, quelle que soit l'appartenance politique du président (Kiewitt, 1983) et n'ont donc pas tendance à punir les administrations démocrates. Ainsi la désaffection des chômeurs vis-à-vis le parti du pouvoir n'est pas automatique. On peut plutôt penser que l'insistance que le chef du Parti libéral a mise sur la question de l'emploi a porté quelques fruits.

\section{Conclusion}

À chacune des élections, entre 1970 et 1981, le Parti québécois a accru ses appuis, tant en chiffres absolus qu'en termes relatifs. Cette progression s'est accompagnée d'une diversification de plus en plus grande de sa clientèle (Blais et Nadeau, 1984). Comme 
le vote péquiste a diminué, tant en chiffres absolus qu'en termes relatifs, entre 1981 et 1985 , on aurait pu s'attendre à un renversement de la tendance à la diversification. Tel n'a manifestement pas été le cas. En particulier, l'électeur péquiste est encore moins typé en 1985 qu'il ne l'était en 1981 par rapport à des caractéristiques comme l'âge et le secteur d'emploi. Non pas que la diversification se soit accentuée: par rapport à la pratique religieuse, par exemple, les électeurs péquistes se démarquent davantage des électeurs nonpéquistes en 1985 qu'en 1981. Il convient plutôt de conclure que la clientèle péquiste apparaît tout aussi hétérogène en 1985 qu'en 1981.

Le fait que le degré de diversification soit relativement stable ne signifie aucunement que les comportements individuels eux, aient été stables. Le tableau 10 donne une indication de l'ampleur des changements. En ne tenant pas compte des mutations entre tiers partis, $25 \%$ des francophones ayant voté en 1981 et en 1985 ont appuyé des partis différents lors de ces deux élections. Il faudrait également considérer ceux qui se sont abstenus à l'une ou l'autre des élections ainsi que les nouveaux électeurs, qui n'avaient pas le droit de vote en 1981; dans notre sondage ces nouveaux électeurs représentent $16 \%$ de l'échantillon.

Les déplacements du vote entre 1981 et 1985 n'ont pas été aléatoires. Le Parti québécois a perdu des appuis tout particulièrement chez la génération née après 1943 et œuvrant dans le secteur public de même que chez les pratiquants réguliers. Le premier groupe constituait un bastion péquiste par excellence et a réagi fortement aux coupures salariales décrétées en 1982. Le second groupe a toujours été peu péquiste mais l'a été encore moins en 1985, possiblement en réaction à la tentative infructueuse du gouvernement péquiste de déconfessionnaliser la structure du système scolaire. On constate ainsi que certaines politiques mises de l'avant pas le Parti québécois ont influencé le comportement électoral de groupes particuliers d'électeurs. C'est là un signe que 
la démocratie électorale fonctionne, les citoyens faisant leur choix électoral tout au moins partiellement à partir de leur évaluation des gestes posés par le gouvernement sortant.

\section{BIBLIOGRAPHIE}

ALDRICK, John H. et Nelson FOREST D., Linear Probability, Loget and Probit Models, (Sage University Paper, Series on Quantitative Applications in the Social Sciences, 07-045, Beverly Hills, Sage, 1984).

BLAIS, A et J. CRÊTE, "Les ménages et le comportement électoral au Québec», texte non publié, 1986.

BLAIS, A., CRÊTE J. et LACHAPELLE, G., (1986), "L'élection québécoise de 1985: un bilan des sondages", Revue canadienne de science politique, 19,2, (juin 1986): 325-336.

BLAIS, André et NADEAU Richard, «L'appui au Parti québécois : évolution de la clientèle de 1970 à 1981 ", pp. 279-318 dans J. Crête (dir.), Comportement électoral au Québec, (Chicoutimi: Gaétan Morin, 1984).

BLAKE, D. E., Political Worlds: Parties and Voting in British Columbia, (Vancouver, U.B.C. Press, 1985).

GINGRAS, F. P. et NEVITTE, N., "La Révolution en plan et le paradigme en cause», Revue canadienne de science politique, 16,4 (décembre 1983): 691-716.

HAMILTON, R. et PINARD, M. (1976), "The Bases of Parti Quebecois Support in Recent Quebec Elections», Revue canadienne de science politique, 9, 1: (mars 1976), 19-26.

JOHNSTON, Richard, "The Reproduction of Religions Cleavages in Canadian Elections», Revue canadienne de science politique, 18,1 (mars 1985): 99-115.

KIEWITT, Roderick D., Maecroeconomics and Micropolitics, (Chicago, University Press, 1982).

LEMIEUX, V., Gilbert M. et BLAIS, A., Une élection de réalignement, (Montréal : Éditions du Jour, 1970).

MADDALA, G. S., Limited dependent and qualitative variables in econometrics, (Cambridge University Press, 1983).

MEISEL, J., Working Papers on Canadian Politics, (Montréal, Mc Gill Queen's Press, 1972).

NADEAU, R., "Modèlisation et analyse empirique des fluctuations de court terme du comportement électoral: le cas canadien ", (projet de thèse, département de science politique, Université de Montréal, Montréal, 1985).

OLZAK, Susan, "Ethnic mobilisation in Quebec", Ethnic and Racial Studies, 5, 3, 1982: 253-275.

PAMMETT, J. H., JENSON, J., CLARKE, H. D., LEDUC, L., "Soutien politique et comportement électoral lors du référendum québécois", pp. 387-419 dans J. CRÊTTE, Comportement électoral au Québec, (Chicoutimi: Gaétan Morin, 1984). 
PERCHERON, Annick, "Religious Affiliation and Political Socialisation in France", West European Politics, 5 (April, 1982): 8-32.

PINARD, Maurice et HAMILTON, "The Independence Issue and the Polarization of the Electorate: The 1973 Quebec Election». Revue canadienne de science politique, 10, 2, (juin 1977): 215-259.

PINARD, M., et HAMILTON, R., (1984), «Les Québécois votent NON: le sens et la portée du vote», pp. 335-385 dans J. Crête (dir.), Comportement électoral au Québec, (Chicoutimi, Gaétan Morin, 1984).

ROSE, Richard, (1974), "Comparability in Electoral Studies", Electoral Behavior: A Comparative Handbook, (New York, The Free Press, 1974).

SCHULZMAN, Kay L. et VERBA, S., Injury to Insult: Unemployment, Class and Political Response, (Cambridge, Harvard University Press, 1979). 